\title{
Research Paper: Effects of Functional Ankle Instability on Balance Recovery Strategy in Athletes
}

\author{
Mohammad Kalantariyan $^{1}$, Hooman Minoonejad ${ }^{1 *}, \operatorname{Reza}_{\text {Rajabi }}{ }^{1}$, Foad Seidi $^{1} \mathbf{Q}$
}

1. Department of Sports Medicine and Health, Faculty of Physical Education and Sport Sciences, University of Tehran, Tehran, Iran.

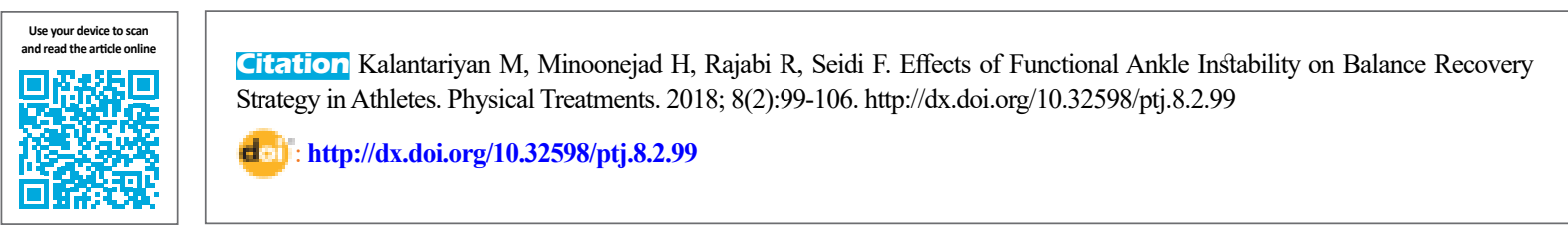



Article info:

Received: 10 Nov 2017

Accepted: 14 Apr 2018

Available Online: 01 Jul 2018

Keywords:

Balance Recovery

Strategy, Functional

Ankle Instability, Athlete,

Jump-landing

\begin{abstract}
A B S T RA C T
Purpose: Many research studies reported balance disorder as one of the most important consequences of Functional Ankle Instability. However, the Balance Recovery Strategy, which its deficiency can cause poor balance or secondary damages, has not been investigated in previous studies yet. The purpose of this article is to study the Balance Recovery Strategy in athletes with Functional Ankle Instability during jumping and landing.
\end{abstract}

Methods: Fifteen male athletes with Functional Ankle Instability and 15 healthy male athletes were randomly assigned into two experimental and control groups, respectively. The subject's Balance Recovery Strategy was observed by Surface Electromyography and during single-foot jumping and landing. In order to evaluate the Balance Recovery Strategy, the ratio of the total activity of the selected hip joint muscles to the total activity of the selected ankle muscles was evaluated. The Multivariate Analysis of Variance (MANOVA) was used to compare the Balance Recovery Strategy between the two groups. Significant level was considered as $\leq 0.05$ in all the statistical analysis.

Results: The results of the statistical tests showed a significant difference between the two groups with regard to the Electromyography activity of femur muscles and ankle muscles. That is, the subjects with Functional Ankle Instability are more likely to use the hip strategy than the ankle strategy to regain their balance. While the group of the healthy subjects used the ankle strategy.

Conclusion: The transfer of the Balance Recovery Strategy from the ankle to the hip joint in the subjects of the Functional Ankle Instability group can be attributed to their proprioception deficit and a decrease in the ankle muscle strength. The reason is that proprioception deficiency can change the motor control programs in the Functional Ankle Instability group.

\footnotetext{
* Corresponding Author:

Hooman Minoonejad, PhD.

Address: Department of Sports Medicine and Health, Faculty of Physical Education and Sport Sciences, University of Tehran, Tehran, Iran

Phone: +98 (912) 3487425

E-mail: h.minoonejad@ut.ac.ir
} 


\section{Highlights}

- Functional ankle instability can change the motor control programs.

- In this situation, the balance recovery strategy is transferred from the ankle to hip due to degradation of proprioception sense and reduces ankle muscle strength.

- This recovery strategy can be considered as an ankle risk factor.

\section{Plain Language Summary}

Functional ankle instability can affect the balance and its correlates, including the balance recovery strategy. This strategy exposes the athlete to various injuries. This issue has not been explored much in previous studies. In this study on athletes with and without functional ankle instability, the hip and ankle joint muscles activities were assessed by surface electromyography during performing single-foot jump-landing tasks. Then, the balance recovery strategy was evaluated by calculating the ratio of hip joint muscle activity to ankle joint muscle activity. According to the results, the athletes with functional ankle instability use their hip muscles more than ankle muscles to maintain and recover their balance compared to normal athletes. This transfer strategy increases the likelihood of loss of balance and injury. This transfer from ankle to hip joint can be attributed to the proprioceptive deficits and thus sending wrong messages from the ankle joint to the nervous system. Over time, this can lead to changes in central nervous system motion control programs. The change in these programs can disrupt the proper activation of lower limb muscles and increase the likelihood of injury.

\section{Introduction}

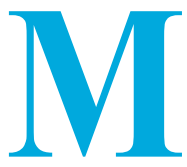

usculoskeletal injuries mostly occur in the ankle. Approximately $25 \%$ of all sports injuries occur in the ankle [1, 2]. Lateral ankle ligament sprains are prevalent in sports such as basketball, volleyball, and handball with frequent jump landings [2]. Functional Ankle Instability (FAI) in athletes is a concerning outcome of the first incidence of lateral ankle sprain in $60 \%$ of all cases [3].

Functional instability refers to the foot's tendency toward recurrent sprains due to the inability to maintain ankle joint stability during dynamic activities such as stair ascent and descent, changing path, or jump landing [4]. FAI can change the neuromuscular function of the nervous system and has adverse consequences [3]. This is because FAI, by disrupting the proprioceptive sense of ankle joint, results in local and general impairments in movement control patterns [5-7].

Chronic pain, osteoarthritis, as well as reductions in the muscle strength around the joint, proprioceptive acuity, and Range of Motion (ROM) of ankle joint dorsiflexion are among local impairments [1, 2]. Furthermore, increased postural sway, balance disturbance, core stability muscles dysfunction, neuromuscular dysfunction of ankle muscles, gait perturbation, kinematics of lower limb proximal joints, and increased reaction time in athletes are among general impairments observed in ankle joint $[5,6,8]$.

According to previous studies, balance is one of the main factors affecting FAI [9-11]. According to Arastoo et al. (2011) people with instability in their ankle joint exhibit balance impairments when performing functional activities. This is because of the impaired function of sensory neurons in these individuals. Such impairment extends from the ankle joint to the central and peripheral nervous system. It also affects the function of motor neurons, leading to the perturbation of postural control while performing dynamic and functional activities [9]. As mentioned before, FAI reduces muscle strength, weakens proprioception sense, and increases the reaction time in athletes. These three factors are highly related to the individuals' balance. Thus, the disruption of these three factors may also affect balance $[6,12,13]$.

In general, humans use different strategies to maintain and recover their balance when faced with sudden perturbations, depending on their severity. They include fixed-support strategies (e.g. ankle and hip strategies where the displacement of the Center of Gravity (COG) is controlled without a change in the base of support) and 
change-in-support strategies (displacement of the COG is controlled with a change in the base of support) [14].

The ankle strategy deflects the trunk's forces in the ankle joint and prevents the transfer of forces to higher joints. Then, it can result in the movement of COG outside the base of support and loss of balance. Therefore, it is the safest strategy when facing postural perturbations $[11,14]$. However, studies report that people with low ankle joint muscle strength, declined proprioception sense and high reaction time usually use hip strategy instead of ankle strategy $[11,12,14]$.

Although the balance recovery strategies have been mainly evaluated in elderly people, few studies have highlighted the importance of balancing strategies in young people and athletes. Riemann stated that patients with unilateral FAI, use hip strategy for postural control, rather than ankle strategy [15]. These people, for compensating the created instability, require higher levels of ankle muscle co-contractions to maintain their ankle stability. However, due to weakness in the ankle muscles, disturbances in the transmission of sensory information, as well as motor information, they have to rely more on hip strategy to correct their movements $[3,15]$.

Therefore, the importance of balance and the strategies applied for its maintenance and recovery is especially higher in athletes who experience more sudden perturbations in functional activities. Thus, the use of ankle strategy seems necessary for this purpose. The available data are scarce on the balancing strategies of non-elderly people. The available studied have focused on comparing these strategies in young people or those with a particular disease. Some researchers also used tools like kinetic or kinematic systems during static activities like singleleg stance $[9,4]$. Therefore, this study aimed to evaluate the Balance Recovery Strategy in young athletes suffering from FAI during single-leg jump landing tasks. We used surface Electromyography (EMG) system.

\section{Materials and Methods}

This was a causal-comparative study. The statistical population consisted of all male athletes in Tehran City, Iran, playing for the university sports teams. The subjects aged 18-25 years and suffered from FAI. The sample size was estimated based on the approaches used in the previous studies [16] and considering the confidence interval of $95 \%$ and a test power of $80 \%$. Based on Mean \pm SD scores reported by Chimera et al. (2004) the sample size was calculated as 12 . Additionally, considering the probability of sample dropouts, it was increased to 15 .
The purposive sampling method was applied to select the study samples from basketball, volleyball, and handball team players. In total, subjects were 15 male athletes with FAI and 15 normal peers (the controls). Those in the FAI group were randomly assigned into three groups of basketball $(n=5)$, volleyball $(n=5)$, and handball $(n=5)$. Subjects in both FAI and control groups were matched on age, height, weight, and Body Mass Index (BMI).

The inclusion criteria were the presence of FAI, a history of at least one lateral ankle sprain injury that needed treatment and inactivity; full weight-bearing ability; normal walking with full ROM of ankle joint; scoring $\leq 27$ from ankle instability questionnaire; no mechanical ankle instability observed by obtaining negative scores from anterior drawer and talar tilt-tests; no pain, weakness, and functional limitation in the affected ankle compared to another ankle; no disorders in nervous or vestibular systems, or head injury; and no histories of participation in any ankle rehabilitation programs over the past 6 months $[17,18]$. On the other hand, the exclusion criteria were unwillingness to participate in the study, feeling of severe pain during the study, and the consumption of any sedatives within 48 hours before the study.

General information about the process of the study was provided to the subjects. Then, they were asked to be present in the laboratory of the Faculty of Physical Education and Sports Sciences of the University of Tehran, at the appointed time. After the presence of subjects in the laboratory, the initial preparations, including wearing suitable sports clothes and shaving excess hair for placing EMG electrodes, acquiring general information and anthropometric measurements, were performed. Next, they were asked to warm-up on a bicycle ergometer for $5 \mathrm{~min}$, then did the lower limb exercises and stretching. Afterward, EMG electrodes were attached. For this purpose, disposable electrodes with a circular contact area of $1 \mathrm{~cm}$ diameter were used.

EMG was demonstrated based on bipolar configuration, where the center-to-center distance was $20 \mathrm{~mm}$ [2]. The determination of electrodes placement was according to the Surface Electromyography for the NonInvasive Assessment of Muscles (SENIAM) standard. Additionally, the exact positions of electrodes were determined by touching the bony landmarks and Maximum Voluntary Isometric Contraction (MVIC) testing. Then, the electrodes were placed on the skin along the muscle fibers [19]. The suitable electrode positions for the muscles were as mentioned below. 
Peroneus longus: one-fourth of the distance from the head of the fibula to the lateral malleolus. The position of the electrode was determined during isometric ankle eversion contractions. Tibialis anterior: one-third of the distance from the tip of the fibula to the inter-malleoli line. The position of the electrode was determined during isometric ankle dorsiflexion contractions. Lateral gastrocnemius: one-third of the distance from the tip of the fibula to the central side of the Achilles tendon. The position of the electrode was determined during isometric ankle plantar-flexion contractions.

Gluteus maximus: half of the distance from the sacrum to the greater trochanter. The position of the electrode was determined during back-lying isometric hip extension. Gluteus medius: half of the distance from the iliac crest to the greater trochanter. The position of the electrode was determined during side-lying isometric hip abduction. Rectus femoris: half of the distance between anterior superior iliac spine and patella. The position of the electrode was determined during back-lying isometric hip flexion $[2,19]$.

After EMG electrodes placement, the subjects were instructed on how to properly perform single-leg jump landing task. Then, they were requested to, first, perform several practice trials to learn more and get prepared. Finally, when the EMG device was recording, each subject was asked to perform the single-leg jump landing task for 5 times, with a 30-second rest between each task.

\section{Single-leg jump landing task}

The lateral ankle sprain injury mainly occurs following dynamic activities like jump landing task. Therefore, the factors associated with this injury should be determined in a similar condition, to find how this damage occurs. Single-leg jump landing task was used in this study because of being a reliable method for analyzing the factors affecting ankle injury. It is also a safe method to challenge ankle stability and can well recover the injury to the ankle sprain $[2,20,21]$.

For performing single-leg jump landing task, as shown in Figure 1, the subject was requested to stand on a 40$\mathrm{cm}$ height platform with hands on the hips. Then, bend the injured foot from the knee while standing on the noninjured foot. The subject was then asked to jump up to $5 \mathrm{~cm}$ and land with the injured foot while maintaining balance for $3 \mathrm{~s}$. The repeatability level of jump height in jump landing task is high. Fitzgerald et al. reported a high Intraclass Correlation Coefficient $(\mathrm{ICC}=0.84)$ for the repeatability level of jump height [22]. The jump landing
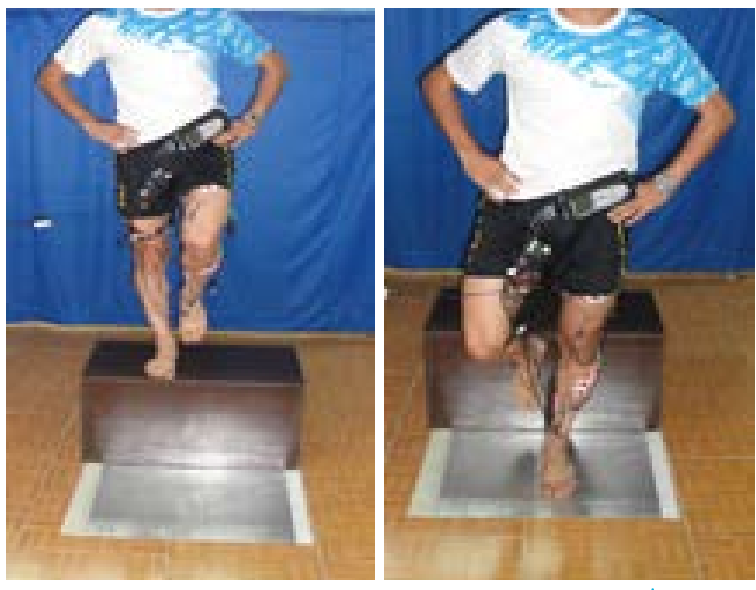

PHYSICAL TREA $\pitchfork$ MENTS

Figure 1. Single-leg jump landing protocol

task was repeated 5 times, and the mean score of 5 correct landings was used to examine the Balance Recovery Strategy. This protocol included standing on the non-injured foot, jumping, and landing with the same foot.

To evaluate the subjects' Balance Recovery Strategy, the ratio of feedback-generated muscle activity of hip joint to the feedback-generated muscle activity of ankle joint (H/A ratio), in a period between initial foot-floor contact and $250 \mathrm{~ms}$ after contact, was calculated. For this purpose, the signals registered at this period were processed in the MegaWin program. In this program, the raw EMG signal was processed by the Root Mean Square (RMS) algorithm with a time constant of $50 \mathrm{~ms}$.

In EMG signals processing, to provide comparability between different muscles and different subjects, muscle activity must be normalized to a reference value [2]. For this purpose, the values obtained from calculating the RMS in muscle activity during perturbations are divided into the values obtained from the MVIC of each muscle. Then, the rate of muscle activity is expressed as a percentage of MVIC (i.e. MVIC \%). Each MVIC test was repeated twice for $5 \mathrm{~s}$, then the average value of $3 \mathrm{~s}$ of MVIC was used to normalize the data.

The study variables were presented by descriptive statistics and the inferential statistics were used to analyze the data in SPSS. To investigate the differences in anthropometric characteristics between the two groups, the Independent t-test was applied. Multivariate Analysis of Variance (MANOVA) was used to compare the Balance Recovery Strategy between the two study groups. The significance level was set at $\mathrm{P}<0.05$. 


\section{Results}

Anthropometric characteristics of the subjects in each study group are presented in Table 1. MANOVA results (Table 2) revealed a significant difference between the groups with respect to the used Balance Recovery Strategy $(\mathrm{P}<0.05)$. Additionally, the FAI group preferred to use hip strategy rather than ankle strategy (Table 3).

\section{Discussion}

The current study evaluated the balance recovery strategies of athletes with FAI during performing jumplanding tasks. The obtained results indicated the use of different balance recovery strategies by athletes with FAI compared to their normal peers. People with FAI, during sudden perturbations like landing after a jump, use muscles of higher joints like the hip joint for compensation.
They use this strategy instead of controlling the perturbations at the ankle joint and preventing it from transferring to larger joints. They are incapable of applying a safe ankle strategy. The findings suggest that the use of an ankle strategy is the safest and most appropriate method to deal with perturbations that disturb the balance [14, 23]. This is because it prevents the transfer of disturbing balance forces to larger joints, avoids the COG from falling outside the base of support, and prevents disturbance of balance and possible damages [14]. This is especially important in athletes who are constantly exposed to sudden perturbations and loss of balance.

Although many studies have investigated the athletes with FAI, and most studies have suggested balance impairment in athletes as one of the most important consequences of the FAI, few research studies have been conducted on their strategy for maintaining and recovering balance. These studies evaluated the Balance Recovery Strategy by assessing static balance with single-leg

Table 1. The independent $t$-test results in respect to the difference between anthropometric characteristics of the groups

\begin{tabular}{|c|c|c|c|}
\hline \multirow{2}{*}{ Variable } & \multicolumn{2}{|c|}{ Mean $\pm S D$} & \multirow{2}{*}{ Sig. } \\
\hline & Control & FAl & \\
\hline Weight, kg & $82.24 \pm 4.51$ & $80.95 \pm 5.17$ & 0.57 \\
\hline Height, cm & $189 \pm 6.12$ & $188 \pm 7.16$ & 0.26 \\
\hline Age, y & $21.7 \pm 2.43$ & $22.01 \pm 3.38$ & 0.39 \\
\hline
\end{tabular}

Table 2. MANOVA results for comparing Balance Recovery Strategy between the study groups

\begin{tabular}{cccc} 
Variable & Wilks' Lambda & df & Sig. \\
\hline Balance Recovery Strategy & 0.237 & 1 & 12.236 \\
\hline & & PHYSICAL TREA MENTS
\end{tabular}

Table 3. MANOVA results for comparing $\mathrm{H} / \mathrm{A}$ ratio between the study groups

\begin{tabular}{|c|c|c|c|c|c|}
\hline \multirow{2}{*}{ Variable } & \multicolumn{2}{|c|}{ Mean $\pm S D$} & \multirow{2}{*}{ df } & \multirow{2}{*}{$\mathbf{F}$} & \multirow{2}{*}{ Sig. } \\
\hline & Control & FAI & & & \\
\hline Average muscle activity of hip joint $(\mathrm{H})$ & $38 \pm 7.38$ & $43 \pm 9.01$ & 1 & 0.646 & 0.55 \\
\hline Average muscle activity of ankle joint (A) & $55 \pm 9.42$ & $36 \pm 6.22$ & 1 & 19.405 & 0.001 \\
\hline \multirow{2}{*}{ Variable } & \multicolumn{2}{|c|}{ H/A } & \multirow{2}{*}{ df } & \multirow{2}{*}{$\mathbf{F}$} & \multirow{2}{*}{ Sig. } \\
\hline & Control & FAI & & & \\
\hline $\mathrm{H} / \mathrm{A}$ ratio (strategy) & $0 / 69$ & $1 / 19$ & 1 & 12.236 & 0.003 \\
\hline
\end{tabular}


stance test. They concluded that the created perturbation was not similar to the natural perturbations that a person experiences on the pitch or during training. Furthermore, the subjects lacked FAI, but only an ankle sprain history $[3,14,15]$. Therefore, this study is the first to investigate the strategy of balance recovery in athletes with FAI and during a completely functional task like jump landing.

The balance recovery approaches include fixed-support strategies like ankle and hip strategies and change-insupport strategies like stepping. Obviously, in fixed-support strategies, the center of mass displacement is much lower and the trunk has more safety. With the higher perturbations, the strategy used for the ankle joint is transferred to larger joints such as hip joint or stepping [13]. However, the strategies used by the trunk to maintain and recover balance can be influenced by various factors. Aging and related physiological changes, certain diseases, vestibular disorders, visual impairment, musculoskeletal abnormalities in the trunk, as well as physical damages are among the most important factors that can change balance strategies from the ankle to hip $[9,13,15]$.

There is a strong association between balance capabilities and balance recovery strategies. FAI is among the most frequent and important factors of balance disturbances [11]. It can disturb the balance and its recovery strategy by reducing muscle strength, degradation of the ankle joint proprioception sense, declining the musculoskeletal system function, and delaying reaction time [7, 15]. Some studies have evaluated the elderly, following muscle weakness, an impaired sense of proprioception, and a slow reaction. They concluded that Balance Recovery Strategy, when confronted with a perturbation, was transferred from the ankle to the hip, which could increase balance problems, and the risk of falling and possible damages $[13,14]$. In people with FAI, like the elderly, ankle muscle weakness, reduced reaction time, as well as declined proprioception sense are evident $[6,7]$.

According to Riemann and Lephart (2002), the high accuracy of proprioception and sensory information received from articulate receptors are important because the nervous system uses sensory information to maintain stability and safety in the joint by sensory triggering either in feedback or feedforward mechanisms [24]. The high accuracy of sensory information, particularly in functional tasks like jump landing which causes high perturbation in the trunk, is more important.

The jump landing task used in the current study challenges the recovery strategy after perturbation in the form of feedback-generated muscle activity. However, the impor- tant role of feedforward activation of muscles in securing stability and avoiding balance disturbance should not be neglected. This is because the central nervous system uses sensory information to regulate muscle stiffness and use predetermined motor programs [25]. Obviously, in patients with FAI, due to the disturbance in ankle joint proprioception sense, the accuracy of sensory information is low. Thus, this contributes to the change of Balance Recovery Strategy from the ankle joint to hip joint, reported by the studied subjects.

According to the obtained results, there was a little difference between the level of hip muscle activity in the healthy and FAI groups (MVIC 38\% vs. MVIC 43\%). However, there was a dramatic difference between them in terms of ankle muscle activity which cause the domination of hip strategy over ankle strategy in the FAI group (MVIC 55\% vs. MVIC 36\%).

It can be concluded that the reason for the change of Balance Recovery Strategy from ankle to hip in people with FAI is the disruption of proper activation of the muscles around the ankle, especially the peroneus longus muscle. The results revealed that ankle injury disturbs the messages transmitted from the mechanical receptors in the ankle joint. Over time, this can reduce proprioception sense by controlling interneurons and reducing the activity of motor units in muscles responsible for controlling ankle movements.

Long-term degradation of proprioception alters reference motor programs and their replacement to inappropriate motor programs with planning errors $[6,24]$. The motor programs controlling ankle movements might be ineffective when faced with sudden perturbations and are unable to activate the ankle muscles properly. In this case, the trunk automatically and by a compensatory mechanism uses other motion control strategies, including hip strategy to maintain balance. The change in using Balance Recovery Strategy from ankle to hip increases the time to achieve stability, during which the COG can move outside the base of support and increase the risk of balance loss and falling [26].

The balance recovery strategies in patients with FAI, in comparison with the controls, have changed from ankle strategy to hip strategy. This can increase the risk of COG's falling outside the base of support, loss of balance, and possible injuries. Therefore, it is recommended that FAI rehabilitation programs be developed to improve the proper functioning of ankle joint muscles when exposed to sudden perturbations. This could also create coordination between the ratio of ankle muscle the activity to hip muscle activity and the ability to restore the Balance Recovery Strategy from the hip joint to ankle joint. Because of cultural issues, 
there were some limitations for evaluating female athletes. Thus, we recommend that further research be conducted on assessing the balance recovery strategies of female athletes with FAI. Moreover, it is suggested that these strategies be evaluated in other sports where the risk of FAI is high and with larger sample size.

\section{Ethical Considerations}

\section{Compliance with ethical guidelines}

This study has been approved by the National Ethics Committee of Biomedical Research (Code: IR.UT.SPORT. REC.1397.025).

\section{Funding}

This research did not receive any specific grant from funding agencies in the public, commercial, or not-forprofit sectors.

\section{Authors contributions}

All authors contributed in preparing this article.

\section{Conflict of interest}

The authors declared no conflict of interest.

\section{References}

[1] Roos KG, Kerr ZY, Mauntel TC, Djoko A, Dompier TP, Wikstrom EA. The epidemiology of lateral ligament complex ankle sprains in National Collegiate Athletic Association sports. The American Journal of Sports Medicine. 2017; 45(1):201-9. [DOI:10.1177/0363546516660980] [PMID]

[2] Kalantariyan M, Minoonejad H, Rajabi R, Beyranvand R Zahiri A. [The comparison of the Electromyography activity of selected muscles of the ankle joint in athletes with ankle dorsiflexion range of motion limitation with healthy athletes during the single-leg jump landing (Persian)]. The Scientific Journal of Rehabilitation Medicine. 2013; 2(2):14-23.

[3] Kazemi K, Arab AM, Abdollahi I, López-López D, CalvoLobo C. Electromiography comparison of distal and proximal lower limb muscle activity patterns during external perturbation in subjects with and without Functional Ankle Instability. Human Movement Science. 2017; 55:211-20. [DOI:10.1016/j.humov.2017.08.013] [PMID]

[4] Clark VM, Burden AM. A 4-week wobble board exercise programme improved muscle onset latency and perceived stability in individuals with a functionally unstable ankle. Physical Therapy in Sport. 2005; 6(4):181-7. [DOI:10.1016/j. ptsp.2005.08.003]
[5] Friel K, McLean N, Myers C, Caceres M. Ipsilateral hip abductor weakness after inversion ankle sprain. Journal of Athletic Training. 2006; 41(1):74-8. [PMID] [PMCID]

[6] Hertel J. Sensorimotor deficits with ankle sprains and chronic ankle instability. Clinics in Sports Medicine. 2008; 27(3):35370. [DOI:10.1016/j.csm.2008.03.006]

[7] Bullock-Saxton JE. Local sensation changes and altered hip muscle function following severe ankle sprain. Physical Therapy. 1994; 74(1):17-28. [PMID]

[8] Gribble PA, Bleakley CM, Caulfield BM, Docherty CL, Fourchet F, Fong DT-P, et al. 2016 consensus statement of the International Ankle Consortium: Prevalence, impact and long-term consequences of lateral ankle sprains. British Journal of Sports Medicine. 2016; 50(24):1493-95. [PMID] [DOI:10.1136/bjsports-2016-096188]

[9] Arastoo AA, Goharpey Sh, Zahednejad Sh, Shaterzadeh Yazdi MJ, Rasouli P. [Effects of star excursion balance training on ankle functional stability via agility hop test in patients with unilateral chronic ankle instability (Persian)]. Jundishapur Scientific Medical Journal. 2011; 10(4):383-93.

[10] Mohammadi A, Shojaedin SS, Letafatkar A, Hadadnejad M. [Comparative effects of impacts and stainabilities of NASM and combined exercises (neuromuscular and core stability) on functional characteristics and balance of individuals with unilateral chronic ankle injury (Persian)]. Scientific Journal of Rehabilitation Medicine. 2018; 7(3):34-47. [DOI:10.22037/ jrm.2017.110665.1442]

[11] Docherty CL, Valovich McLeod TC, Shultz SJ. Postural control deficits in participants with Functional Ankle Instability as measured by the balance error scoring system. Clinical Journal of Sport Medicine. 2006; 16(3):203-8. [PMID]

[12] Wikstrom EA, Naik S, Lodha N, Cauraugh JH. Balance capabilities after lateral ankle trauma and intervention: A meta-analysis. Medicine \& Science in Sports \& Exercise. 2009; 41(6):1287-95. [DOI:10.1249/MSS.0b013e318196cbc6] [PMID]

[13] Mackey DC, Robinovitch SN. Mechanisms underlying agerelated differences in ability to recover balance with the ankle strategy. Gait \& posture. 2006; 23(1):59-68. [DOI:10.1016/j. gaitpost.2004.11.009] [PMID]

[14] Beyranvand R, Sahebozamani M, Daneshjoo A. [The effect of hyperkyphosis on Balance Recovery Strategy of young and old people in response to sudden perturbations (Persian)]. Bimonthly Official Publication Daneshvar Medicine. 2017; 24(130):37-47.

[15] Riemann BL. Is there a link between chronic ankle instability and postural instability. Journal of Athletic Training. 2002; 37(4):386-93. [PMID] [PMCID]

[16] Chimera NJ, Swanik KA, Swanik CB, Straub SJ. Effects of plyometric training on muscle-activation strategies and performance in female athletes. Journal of Athletic Training. 2004; 39(1):24-31. [PMID] [PMCID]

[17] Delahunt E, Coughlan GF, Caulfield B, Nightingale EJ, Lin CWC, Hiller CE. Inclusion criteria when investigating insufficiencies in chronic ankle instability. Medicine \& Science in Sports \& Exercise. 2010; 42(11):2106-21. [DOI:10.1249/ MSS.0b013e3181de7a8a] [PMID]

[18] Samadi H, Rajabi R, Karimizadeh Ardakani M. The effect of six weeks of neuromuscular training on joint position sense 
and lower extremity function in male athletes with Functional Ankle Instability (Persian)]. Journal of Sport Medicine. 2017; 9(1):15-33. [DOI:10.22059/JSMED.2017.62869]

[19] Hermens HJ, Freriks B, Merletti R, Stegeman D, Blok J, Rau $\mathrm{G}$, et al. European recommendations for surface Electromyography. Roessingh Research and Development. 1999; 8(2):13-54. [PMID]

[20] Suda EY, Amorim CF, Sacco ID. Influence of ankle functional instability on the ankle Electromyography during landing after volleyball blocking. Journal of Electromyography and kinesiology. 2009; 19(2):e84-93. [DOI:10.1016/j. jelekin.2007.10.007]

[21] Caulfield B, Crammond T, O'Sullivan A, Reynolds S, Ward T. Altered ankle-muscle activation during jump landing in participants with functional instability of the ankle joint. Journal of Sport Rehabilitation. 2004; 13(3):189-200.

[22] Fitzgerald D, Trakarnratanakul N, Smyth B, Caulfield B. Effects of a wobble board-based therapeutic exergaming system for balance training on dynamic postural stability and intrinsic motivation levels. Journal of Orthopaedic \& Sports Physical Therapy. 2010; 40(1):11-9. [DOI:10.2519/ jospt.2010.3121] [PMID]

[23] Feger MA, Donovan L, Hart JM, Hertel J. Lower extremity muscle activation during functional exercises in patients with and without chronic ankle instability. PM\&R. 2014; 6(7):602-11. [DOI:10.1016/j.pmrj.2013.12.013] [PMID]

[24] Riemann BL, Lephart SM. The sensorimotor system, part II: The role of proprioception in motor control and functional joint stability. Journal of Athletic Training. 2002; 37(1):80-4. [PMID] [PMCID]

[25] Riemann BL, Myers JB, Lephart SM. Sensorimotor system measurement techniques. Journal of Athletic Training. 2002; 37(1):80-4. [PMID] [PMCID]

[26] Shaw MY, Gribble PA, Frye JL. Ankle bracing, fatigue, and time to stabilization in collegiate volleyball athletes. Journal of Athletic Training. 2008; 43(2):164-71. [PMID] [PMCID] 